\title{
Novel function of stabilin-2 in myoblast fusion: the recognition of extracellular phosphatidylserine as a "fuse-me" signal
}

\author{
Go-Woon Kim ${ }^{1}$, Seung-Yoon Park ${ }^{1,2} \mathcal{E}$ In-San Kim ${ }^{1,3, *}$ \\ ${ }^{1}$ Center for Theragnosis, Biomedical Research Institute, Korea Institute of Science and Technology (KIST), Seoul 02792, ${ }^{2}$ Department of \\ Biochemistry, School of Medicine, Dongguk University, Gyeongju 38066, ${ }^{3}$ KU-KIST School, Korea University, Seoul 02841, Korea
}

\begin{abstract}
Myoblast fusion is important for skeletal muscle formation. Even though the knowledge of myoblast fusion mechanism has accumulated over the years, the initial signal of fusion is yet to be elucidated. Our study reveals the novel function of a phosphatidylserine (PS) receptor, stabilin-2 (Stab2), in the modulation of myoblast fusion, through the recognition of PS exposed on myoblasts. During differentiation of myoblasts, Stab2 expression is higher than other PS receptors and is controlled by calcineurin/NFAT signaling on myoblasts. The forced expression of Stab2 results in an increase in myoblast fusion; genetic ablation of Stab2 in mice causes a reduction in muscle size, as a result of impaired myoblast fusion. After muscle injury, muscle regeneration is impaired in Stab2deficient mice, resulting in small myofibers with fewer nuclei, which is due to reduction of fusion rather than defection of myoblast differentiation. The fusion-promoting role of Stab2 is dependent on its PS-binding motif, and the blocking of PS-Stab2 binding impairs cell-cell fusion on myoblasts. Given our previous finding that Stab2 recognizes PS exposed on apoptotic cells for sensing as an "eat-me" signal, we propose that PS-Stab2 binding is required for sensing of a "fuse-me" signal as the initial signal of myoblast fusion. [BMB Reports 2016; 49(6): 303-304]
\end{abstract}

\section{*Corresponding author. E-mail: iskim14@kist.re.kr \\ http://dx.doi.org/10.5483/BMBRep.2016.49.6.078}

Received 7 May 2016

Keywords: Muscle regeneration, Myoblast fusion, Phosphatidylserine, Stabilin-2

Abbreviations: Stab2, stabilin-2; PS, phosphatidylserine

Perspective to: Seung-Yoon Park et al (2016), Stabilin-2 modulates the efficiency of myoblast fusion during myogenic differentiation and muscle regeneration, Nature Communications, 7:10871, doi: $10.1038 /$ ncomms 10871
Skeletal muscle is formed from myogenic precursors. After the initial myogenic commitment, mono-nucleated myoblasts fuse with each other and form multinucleated myofibers. For the past two decades, numerous molecules have been suggested as regulators of myoblast fusion, including membrane receptors, transcription factors, and signaling molecules. However, the details of fusion mechanism has been elusive.

Phosphatidylserine (PS) is one of the phospholipid membrane components and is normally localized at the inner leaflet of the plasma membrane. Exposure of PS to the outer plasma membrane has important roles in various cellular events, such as an "eat-me" signal for apoptotic cell clearance, a scaffold for coagulation factors in activated platelets, and a candidate signal for cell-cell fusion. In the case of myoblast fusion, the function of PS exposure has not been extensively analyzed. However, it is known that the transient exposure of PS on the extracellular membrane of fusing myoblasts is needed for myoblast fusion. A recent study showed that a PS-receptor, brain-specific angiogenesis inhibitor 1 (Bai1), recognized the PS exposed on apoptotic cells and promoted fusion between healthy myoblasts, which may result in the recruitment of ELMO/Rac1 (Hochreiter-Hufford et al. (2013) Nature 497: 263-267, doi:10.1038/nature12135). However, the receptor recognizing PS on healthy myoblasts has not been identified.

Our study has reported for the first time that the function of Stab2 in myoblast fusion is related to the recognition of PS exposed in healthy myoblasts. The expression level of Stab2 was higher than other PS receptors on mouse skeletal muscles, and increased depending on the differentiation days of $\mathrm{C} 2 \mathrm{C} 12$ and primary myoblasts. Stab2 expression was regulated by calcineurin/NFAT signaling; specifically, the NFATc1 binding to the Stab2 promoter region enhanced the expression of Stab2 transcript. The forced expression of Stab2 increased the fusion of $\mathrm{C} 2 \mathrm{C} 12$ cells, but there were no differences in the expression of myogenin, one of the differentiation makers for committed myoblasts. Furthermore, mouse fibroblasts L cells, which usually do not fuse with each other, acquired fusogenic activity after the forced expression of Stab2, suggesting the role of Stab2 in the positive regulation of cell-cell fusion.

Stab2-deficient mice exhibited a decrease in skeletal muscle

ISSN: 1976-670X (electronic edition)

Copyright (c) 2016 by the The Korean Society for Biochemistry and Molecular Biology

(ㄷ) This is an open-access article distributed under the terms of the Creative Commons Attribution Non-Commercial License (http://creativecommons.org/licenses/by-nc/4.0) which permits unrestricted non-commercial use, distribution, and reproduction in any medium, provided the original work is properly cited. 
weight relative to body weight, which was due to the reduction of myofiber size rather than changes in the number of myofibers. Myonuclei number per myofiber was decreased in Stab2-deficient muscle compared with wild-type muscle, indicating defective myoblast fusion by Stab2 deficiency. Consistent with this, the fusion of Stab2-deficient myoblasts was lesser than that of wild-type myoblasts. Ablation of Stab2 caused deterioration of muscle regeneration after muscle injury; the regenerating muscles of mice lacking the Stab2 gene contained smaller myofibers and fewer centralized nuclei. However, proliferation and activation of satellite cells during muscle regeneration was not affected by Stab2 ablation, which implied the role of Stab2 in myoblast fusion, but not in myogenic differentiation.

Other than as a PS receptor, Stab2 has another function as a scavenger receptor for hyaluronic acid and chondroitin sulfates. We found that a significant reduction of myoblast fusion was shown by the sole treatment of EGF-like domain repeat for PS binding and not the Link domain for hyaluronic acid and chondroitin sulfates, indicating the role of Stab2 as a PS-receptor in myoblast fusion. The blocking of external PS using anti-PS antibodies reduced myoblast fusion on wild-type myoblasts, while it did not change the fusion index on Stab2-deficient myoblasts. Besides, in contrast to promotion of wild-type myoblast fusion by PS/PC liposomes, treatment with PS/PC liposomes did not raise the fusion index of Stab2 deficient myoblast. These evidences suggested that the fusion-increasing activity of Stab2 was reasonably related with its function in recognition of PS exposed in myoblasts. Furthermore, excess myonuclear accretion in $\mathrm{C} 2 \mathrm{C} 12 / \mathrm{Stab} 2$ cells was abolished by anti-PS antibodies but not by caspase inhibitors, indicating that Stab2 promotes myoblast fusion by interacting with PS exposed on live myoblasts rather than apoptotic myoblasts.

Stab2 also acts as a receptor for apoptotic cell clearance (efferocytosis). After recognizing PS exposed on apoptotic cells, Stab2 activates the signaling cascades through both Gulp/Rac1 and ELMO/DOCK180/Rac1 pathways, thereby inducing actin polymerization for making the phagocytic cup (Kim et al. (2012) Mol. Cell. Biol. 32(14):2698-2708, doi: 10.1128/MCB.06743-11). In fact, actin cytoskeleton regulator proteins, such as ELMO/Dock1, Arf-6 and N-WASP, function for myoblast fusion as well as efferocytosis. Also, as stated in a recent study of $C$. elegans, the phagocytic pathway components, including phosphatidylserine receptor (PSR-1), are also needed for regenerative axonal fusion (Neumann et al. (2015) Nature 517:219-222, doi:10.1038/nature14102). Therefore, this raised the possibility that the signal from Stab2-PS binding can recruit fusion-mediating molecules and proceed to myoblast fusion. In comparison with defects observed in Rac and Myomaker deficiencies, mild fusion phenotypes in Stab2-deficient mice may be explained by redundancy of signaling pathways. In our perspective, Stab2 and PS binding may work as a signaling cascade for "fuse-me" by recruitment of fusion mediating proteins during formation of myofibers.

\section{ACKNOWLEDGEMENTS}

This study was supported by the KIST Institutional Program (Project No. 2E26320). 